\title{
Preparing an AIDS policy for colleges of osteopathic medicine
}

\author{
WILLIAM F. DUERFELDT, DO
}

There are currently 1.5 million people in the United States infected with the human immunodeficiency virus. In the next five to ten years, this nation's health care facilities and medical schools could be inundated with staggering numbers of the sick and dying. Unless our osteopathic and allopathic colleges and allied health care centers are adequately prepared for this crisis, many of these people will not receive adequate care. A recent survey conducted by Ohio University College of Osteopathic Medicine (OU-COM) revealed that many osteopathic colleges do not have a formal policy to deal with the multiple complex issues of the AIDS epidemic. This brief article reviews the approach which OUCOM took to correct this deficiency. In addition, the article includes OU-COM's conclusions and recommendations regarding many of the salient issues, with a discussion of the rationale benind those conclusions.

There currently are 1.5 million people infected with human immunodeficiency virus (HIV) in the United States. ${ }^{1}$ Although the hypothesis is not confirmed, it appears that all persons now infected with HIV eventually will succumb to AIDS. ${ }^{2}$ In the next five to ten years, this nation's physicians, hospitals, health care centers, and medical training centers will be confronted with a staggering number of sick and dying AIDS patients.

During the coming decade, academic health centers will be at the center of the AIDS epidemic because of research and the volume of patients they will treat. Concern over this fact is mounting among students and residents. Even attending staff and faculty, as well as medical center and hospital leaders, frequently are reluctant to encourage any expansion of HIV-related cases because of their concern about the infection risk. ${ }^{3}$ In addition, there is growing uneasiness among health professionals over their own safety and that of their families, especially as the probability of coming into contact with an HIV-positive patient increases. ${ }^{3}$ Further, according to the Associated Medical Schools of New York, ${ }^{3}$ "The association of HIV-related illnesses with both homosexuality and intravenous drug users (Fig 1) bring to the fore many preju-

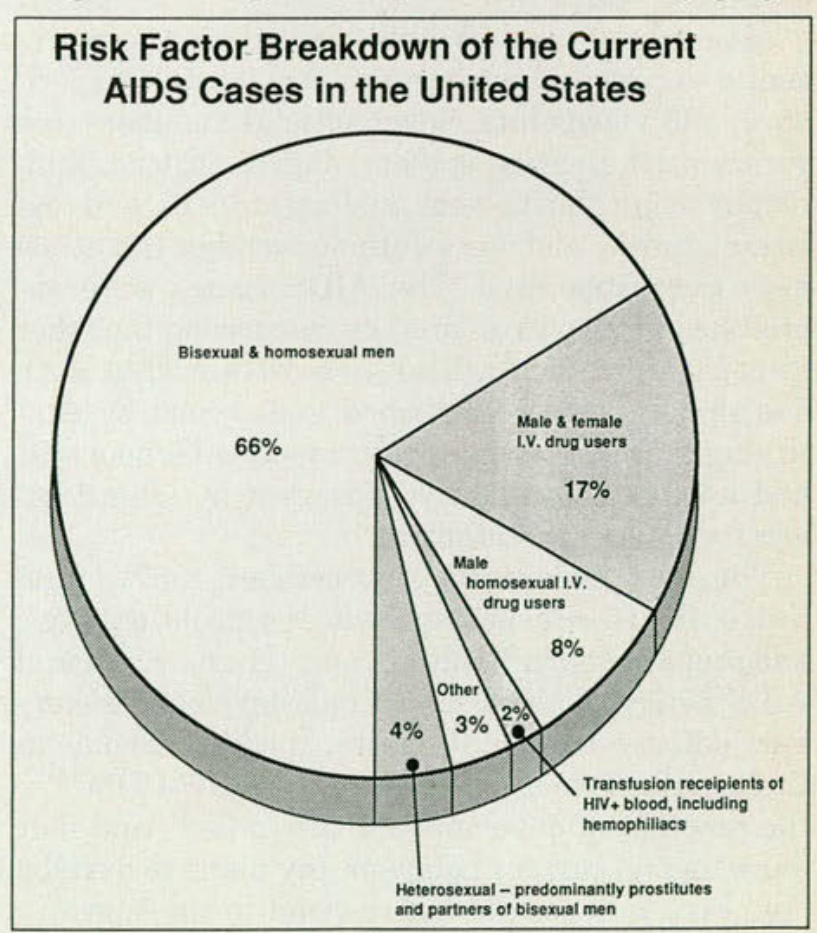

Fig 1. Risk factor analysis of the current AIDS cases in the United States. 
dices, from which the health professions are not exempt."

Is the osteopathic medical profession ready to deal with this crisis? Are the nation's osteopathic medical training centers preparing themselves to cope with the multifaceted issues presented by this spreading epidemic? It is imperative that every osteopathic medical school in the nation prepare a plan of action in the form of a carefully considered AIDS policy designed to address the issues particular to their institution.

\section{Response by Ohio University College of Osteopathic Medicine (OU-COM)}

Early in 1987, the Office of Health, Education and Wellness (Student Health Services) of Ohio University published a [General Statement on Ohio University's Response to AIDS.] This publication was helpful in providing the general student body and faculty with background information regarding the epidemiology, risk factors, and the university's legal response to the AIDS crisis.

It did not, however, address the multiple and often complex issues particular to a medical school and its allied health care centers, such as OUCOM and the Osteopathic Medical Center (OMC). Therefore, in October 1987, the dean of OU-COM appointed an AIDS Task Force. Its primary mission was to consider the legal, ethical, and medical problems associated with the AIDS epidemic and to formulate a comprehensive AIDS policy for OUCOM and OMC.

Members of the task force were chosen to represent a variety of backgrounds, knowledge, experience, and viewpoints. Seven official members (basic scientist, dentist, epidemiologist, ethicist, family physician, fourth-year medical student, and registered nurse) and one ex officio member (an attorney) were appointed. The AIDS issues were approached in two ways: first, by canvassing the other osteopathic medical schools to ascertain what work had already been accomplished; and, second, by identifying those issues unique to a medical school that had not been previously addressed by Ohio University's general statement.

The task force survey (December 1987) found that of the 15 osteopathic medical schools, only two had prepared an individual, comprehensive, formal AIDS policy. A third school had developed a common policy with the allopathic medical schools in their state. Two colleges were, like OU-COM, "in the process" of developing such a policy, and four more had no current policy or any plans to develop one. Five colleges did not respond to the survey.

Five issues were identified as being particularly relevant to every osteopathic college; these included mandatory HIV testing, confidentiality, the obligation of student and faculty participation in the care of HIV-positive patients, patient, student, and faculty education, and measures to prevent transmission of HIV.

\section{The policy}

In keeping with the format of Ohio University's general statement and to enhance the ease with which the policy could be reviewed, the task force used a question and answer style. The material was categorized into five sections-general, employee, student, and patient guidelines, and HIV testing. In addition, the complete lft2lMMWR supplement, "Recommendations for prevention of HIV transmission in health-care settings," ${ }^{4}$ was included as an appendix.

Copies of the entire policy are made available to every department and departmental section of OU-COM and OMC on the Athens campus. Additional copies are given to all individual personnel requesting them.

Further, a shortened version (summary) has been distributed to all OU-COM medical students prior to their initial clinical rotations. Beginning with the first year class of 1988 , the summary also is provided to every incoming first-year student. Finally, an AIDS question and answer session has been scheduled as a regular feature of the phase III (preclinical) curriculum. This allows students an opportunity to comprehend the nature of the current crisis, their roles as medical students in the delivery of health care to AIDS, ARC, and HIVpositive patients, and the critical importance of practicing preventive measures to reduce their own risk and that of their patients.

\section{Recommendations}

The conclusions and recommendations of the OUCOM AIDS Task Force regarding some of the salient issues follow. In addition, the rationale behind a decision, where the issue may be controversial or require further explanation, also is provided. (The AIDS policy in its entirety is available upon request.)

(1) Medical faculty, residents, medical students, and all health care workers associated with $O U$ COM or OMC will accept as their most fundamental responsibility the care of all patients who come before them. (In this regard, OU-COM is in full and absolute agreement with the 1987 statement published by the Associated Medical Schools of New York $^{3}$ : "The treatment of AIDS is the function of the doctor's mission; the doctor's mission cannot 
be changed because of the small but real dangers inherent in treating AIDS. Communicable diseases have traditionally called for sacrifice and courage from the medical community. In addition, medical schools and academic health centers have a special duty to fight not only the disease itself but the fear and misinformation surrounding the disease.")

(2) Mandatory HIV testing of students and employees shall not be required. (The specificity of the combined ELISA and Western Blot tests is about $99 \%{ }^{5}$ This means that for each 500 persons tested, three would be labeled falsely as HIV positive. Minimally, these persons would suffer severe psychologic trauma and might face loss of educational opportunities, health insurance, family support, job, or housing. HIV is transmitted by the transfer of infectious material from the bodily fluids of an infected individual into the body of a susceptible individual. Health care workers frequently are exposed to body fluids of patients. The converse, exposure of a patient to body fluids of a health care worker, seldom occurs. While we are unaware of any statistics based on observations of patients exposed to HIV-positive care givers, there are extensive data on health care workers exposed to HIVpositive patients and their body fluids. To date, over 61,000 cases of AIDS have been diagnosed, and approximately 25,000 AIDS patients have died. These patients have received care from thousands of health care workers over the past 10 years - care that frequently exposed these providers to infectious body fluids. Out of these thousands of personyears of exposure, only a half-dozen health care workers are documented to have acquired HIV infection from patients. The risk, while measurable, is quite small-less than the risk of acquiring a fatal case of hepatitis B, for example. The risk of HIV transmission from an HIV-positive health care worker to a susceptible patient would be even less. The implementation of the recent CDC "Guidelines for preventive AIDS in health care workers" will reduce the risk even further for patients as well as for health care workers themselves. ${ }^{4}$ These guidelines already have been adopted by OU-COM/OMC. The task force concluded, therefore, that the risk of a patient acquiring an infection from an HIVpositive employee of OU-COM/OMC is negligible and would not be altered substantially by a program of mandatory testing.)

(3) The right to confidentiality is paramount to the person with $A I D S$. (When a person with AIDS comes to the attention of OU-COM, whether patient, student, or employee, the Director of Medical Services will conduct a thorough review based on the best medical and legal information available. Any actions taken will respect the confidentiality and welfare of the individual as well as that of Ohio University, OU-COM/OMC, including its students, patients, and employees, and will be in compliance with all CDC guidelines and state and federal regulations.)

(4) The fact that a classmate or fellow employee has AIDS or ARC or is HIV positive will not be a legitimate reason for a student or employee to seek reassignment.

(5) Employees, faculty, and students working in areas where they can be exposed to body fluids or blood spills should follow general housekeeping procedures and take precautions as recommended by the $C D C$. (Equipment or services exposed to blood or other body fluids should be cleansed with a germicidal agent such as SOAK, A-500, or a 1:10 dilution of household bleach. Employees should use protective gloves, surgical masks, eye protectors, and effective barrier gowns as recommended by the $\mathrm{CDC}^{4}$ whenever the chance of exposure to open skin or mucous membranes is present.)

(6) Any physician or student who has reason to believe that he or she may be a carrier of the HIV virus bears the responsibility of limiting his or her practice to noninvasive care and using appropriate barrier techniques and sterilization procedures in the course of all patient contact. (This is in keeping with the policy adopted by the Ohio State Medical Board. ${ }^{6}$ The task force slightly modified the $M M W R$ definition 4 and defined "invasive procedures" as "... any entry into tissue, cavities, or organs, or repair of major traumatic injuries such as (1) in an operating or delivery room, emergency department, or outpatient setting, including both physicians' and dentists' offices; (2) drawing blood, starting an intravenous line, or giving an injection; (3) cardiac catheterization and angiographic procedures; (4) a vaginal or cesarean delivery or other invasive obstetric procedure during which bleeding may occur; (5) the manipulation, cutting, or removal of any oral or perioral tissues, including tooth structure, during which bleeding occurs or the potential for bleeding exists; or (6) endoscopy procedures, such as esophagogastroduodenoscopy, bronchoscopy, sigmoidoscopy, etc. Decisions regarding activities of an HIV-infected medical student should be consistent with the established policies governing physicians. If a medical student suspects or confirms that he or she is HIV-positive or has ARC or AIDS, that student should report this information to the Associate Dean for Academic and Clinical Affairs. Whether the medical student reports it, or such knowledge is acquired through 
TABLE 1. INFORMED CONSENT FORM FOR HIV ANTIBODY TEST.

I hereby give my consent to have my/my child's blood tested to find out whether or not I/my child have antibodies to HIV in my blood. Doctors think that HIV causes Acquired Immune Deficiency Syndrome (AIDS). The reason my doctor is recommending this test is one or more of the following:

1. I/my child may have been intimate with someone with AIDS.

2. I/my child may have received blood from someone with AIDS.

3. I am pregnant and may have been intimate or received blood from someone with AIDS.

4. Other situations such as tuberculosis, recipient of a skin graft, or

(write in reason)

I have been informed that when a person is infected with a virus, his/her body produces antibodies that fight the infection. If $I / m y$ child has been infected with HIV, my body may be producing antibodies to this virus. This blood test will determine whether I/my child has HIV antibodies. If these antibodies are present, it means that $\mathrm{I} / \mathrm{my}$ child might be contagious for others, or, if I am pregnant, my unborn baby could be infected. On the other hand, I have been told that a positive test does not necessarily mean I will get sick with AIDS even though I may be contagious.

I have been informed that sometimes the test result may be negative even though I may have been infected with the virus (false negative). This can happen because it takes time for the body to make antibodies and there may not have been enough time for the antibodies to appear before the test is performed. I have been informed that sometimes the test can indicate that a person has antibodies to the virus when that person is not really infected (false positive). In order to reduce this risk, positive tests will be retested.

I understand that the results of this blood test will become a part of $\mathrm{my} / \mathrm{my}$ child's permanent medical record and will be released to health practitioners responsible for $\mathrm{my} / \mathrm{my}$ child's care and treatment. Efforts will be made to keep the results of this test confidential and the University will not release confidential or medical information except as required by law. While I understand that it may be necessary to release this information to others who may pay for my/my child's health care, I further understand that this will require my signature on a separate release form.

The consent form has been explained to me and all questions regarding the nature of the blood test, its expected benefits, its risks, and alternative tests have been answered to my satisfaction. I understand that I may ask additional questions at any time.

Signature: Date:

Physician: Date: other means, a thorough and complete evaluation will be conducted by the Associate Dean for Academic and Clinical Affairs and the Committee on Student Progress. This committee, which has as one of its responsibilities approval of alterations and course of study, will consider the effects of removing or excusing the student from direct patient contact involving invasive procedures and will make a recommendation to the dean as to whether the student, if excused, can complete the course of study that will meet the requirements for graduation as an osteopathic physician. The final decision regarding the student's completion of medical school will rest with the dean.)

\section{HIV testing recommendations}

Screening. If a faculty member, student, or employee has a concern relative to AIDS, he or she may consult one of the OU-OMC physicians for further information. If a student desires routine screening for HIV antibody but is considered to be at low risk, he/she will be referred to one of several authorized AIDS testing centers for confidential testing and counseling.

Diagnostic evaluation. If a student feels he/she has AIDS or ARC because of symptoms or known exposure and consults an OU-OMC physician, an appropriate medical evaluation will be performed. The OMC Medical Director or other designated physician will provide confidential counseling and any further indicated medical follow-up based on CDC guidelines. The patient's right to confidentiality is paramount. OU-OMC physicians will not release confidential medical information except as required by law or OU-COM policy.

Consent form. An informed consent form (Table 1) is available for any OU-OMC physician who wishes to have a patient tested for the HIV antibody. This form explains the possible reasons for the test and should be signed by both the patient and the physician. The results of this test will become a permanent part of the patient's OU-OMC chart.

\section{Conclusions}

The experience of the health care systems in the United States and throughout the world suggest that if there are not sufficient funds, resources, and preparation for treatment and care of persons with AIDS, they will not receive care. ${ }^{1}$ Our survey suggests that osteopathic medical schools and their allied health care centers are unprepared to deal with the AIDS crisis. It is the purpose of this article to 


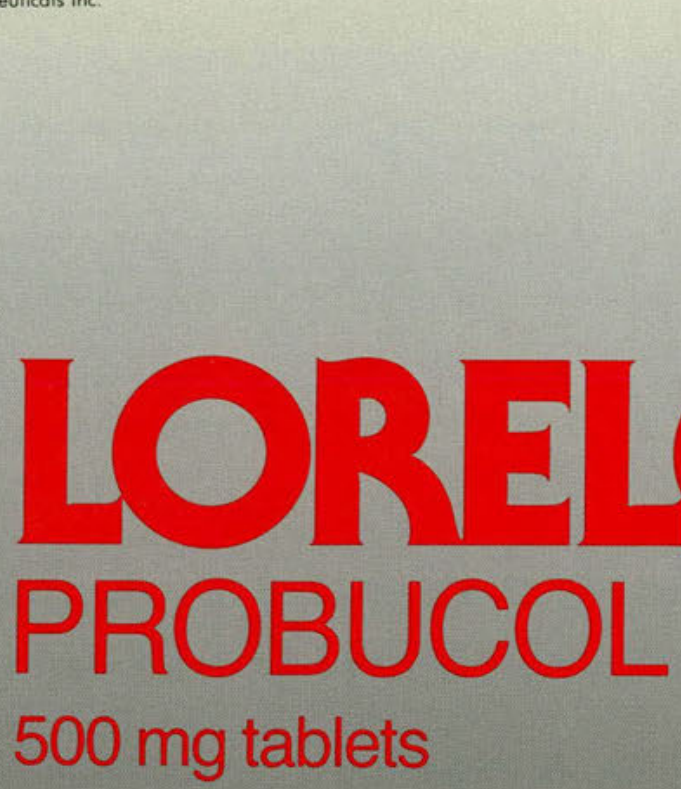

as an adjunct to diet

\section{Now, double strength tablets}

- dependable efficacy - lowers cholesterol by up to $27 \%$

- helps ensure excellent patient compliance

- has a well-established side effects profile*

- costs significantly less than the resins

\section{For continued, convenient b.i.d. dosage}

relco is indicated for the reduction of evated serum cholesterol in patients with imary hypercholesterolemia, as an junct to diet.

\section{errell Dow U.S.A.}

\section{son of Mereel Dow phamascoutcals sin}

relco is not an innocuous drug and strict tention should be paid to the Indications, ontraindications, Warnings, and Precautions.
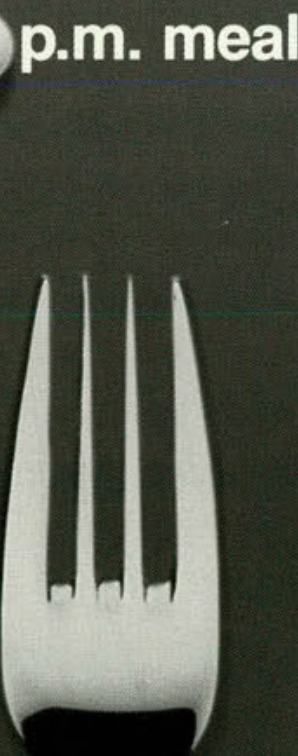

(R)
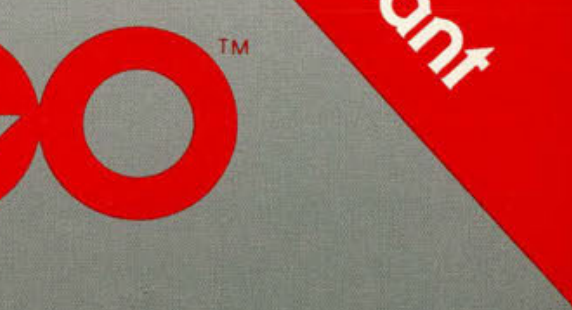


\section{Lorelco ${ }^{\circledR}$ Tablets (probucol)}

\section{CAUTION: Federal law prohibits dispensing without prescription.}

DESCRIPTION: Lorelco (probucol) film-coated tablets for oral administration contain $250 \mathrm{mg}$ or $500 \mathrm{mg}$ of probucol per hydroxypropyl methylicellulose 2910 , iron oxide, lactose, magnesium stearate, microcrystaline celliulose, polysorbate 80 , talc, and titanium dioxide. Lorelco is an agent for the reduction of elevated serum cholesterol. The chemical name is $4.4^{\prime}-1\left(11^{-m e t h y l}\right.$ thylidene) bis(thio) bis [2,6-bis(1,1-dimethyl. resemble that of any other available cho

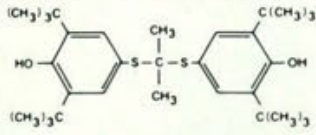

CLINICAL PHARMACOLOGY: Lorelco lowers total serum cholesterol and has relatively little effect on serum triglycerides

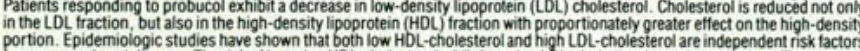

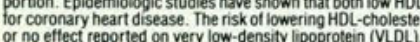

Studies on the mode of action of Lorelco indicate that it increases the fractional rate of $L O L$ catabolism. This effect may be linked orelco also exhibits inhibition of early stages of cholesterol biosn thesis and slight inhibition of absorption of diecy. cholesterol. There is no increase in the cyclic precursors of cholesterol namely desmosterol and 7-dehydrocholesterol. On this basis, it is concluded that Lorelco does not affect the later stages of cholesterol biosynthesis.

Absorption of Lorelco from the gastrointestinal tract is limited and variable. When it is administered with food, peak blood levels
are higher and less variable. With continuous administration in a dosage of $500 \mathrm{mg}$ b.i.d., the blood levels of an individual gradually increase over the first three to four months and thereatter remain tairly constant. in 116 patients treated with Loreico $062.0 \mathrm{mcg} / \mathrm{mL}$. In a separate study in eight patients, blood levels averaged $19.0 \mathrm{mcg} / \mathrm{mL}$ a t the end of $12 \mathrm{mLonths}$ of treatment Six weeks after cessation of therapy, the average had tallen by $60 \%$. After six. months, the average had tallen by $80 \%$ In December 1984, a National Institutes of Health Consensus Development Conference Panell concluded that lowering definitely elevated blood cholesterol levels (specifically blood levels of LDL-cholesterol) will reduce the risk of heart attacks due to

INDICATIONS AND USAGE: Serious animal toxicity has been encountered with probucol. See WARNINGS and ANIMAL INDICATIONS, CONTRAINDICATIONS, and WARNINGS Drug therapy should not be used for the routine treatment of elevated blood lipids for the prevention of coronary heart disease Dietary therapy specific for the type of hyperlipidemia is the initial treatment of choice. Excess body weight may be an importan
tactor and should be addressed prior to any drug therapy. Physical exercise can be an important ancillary measure. Contributory
disease such as hyypothyroidism or diabetes mellitus should be looked tor and adequately treated. The use of drugs should be disease such as hypothyroidism or diabetes mellitus should be looked for and adequatery treated. The use of drugs should be ultimately is to use drugs, the patient should be instructed that this does not reduce the importance of adhering to diet

The selection of patients for cholesterol-lowering drug therapy should take into account other important coronary risk factors such as smoking, hypertension, and diabetes melitus. Consideration should be given to the efficacy, safety. and compliance
factors for each of the cholesterol-lowering drugs prior to selecting the one most appropriate for an individual patient. Lorelco may be indicated for the reduction of elevated serum cholesterol in patients with primary hypercholesterolemia (Types

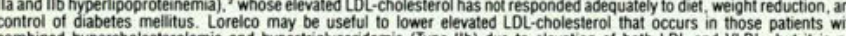
combined hypercholesterolemia and hypertriglyceridemia (Type IIb) due to elevation of both LDL and VLDL, but it is no: cholesterol represents a primary lipid disorder, it should be determined that patients being considered for treatment with Lorelco
have an elevated LDL cholesterol as the cause for an elevated total serum cholestero. This may be particularly relevant for patients with elevated triglycerides or with markedly elevated HDL-cholesterol values, where non-LDL fractions may contribute
significantly to total cholesterol levels without apparent increase in cardiovascular risk. In most patients, LOL-cholesterol may
be estimated according to the following equation:

$$
\text { LDL-cholesterol }=\text { Total cholesterol }-\lfloor(0.16 \times \text { triglycerides })+\text { HDL-cholesterol }]
$$

When total triglycerides are greater than $400 \mathrm{mg} / \mathrm{dL}$, this equation is less accurate. In such patients. LDL-cholesterol may be obtained by ultracentrifugation.

It is not always possible to predict from the lipoprotein type or other factors which patients will exhibit tavorable results. Lipid

The effect of probucol-induced reduction of serum cholesterol or triglyceride levels, or reduction of HDL-cholesterol levels on CONTRAINDICATIONS: (See also WARNINGS and PRECAUTIONS.) Lorelco is contraindicated in patients who are known to ave a hypersensitivity to it. Lorelco is contraindicated in patients with evidence of recent or progressive myocardial damage of findings suggestive of serious ventricular arthythmias or with unexplained syncope or syn

WARNINGS: SERIOUS ANIMAL TOXICITY HAS BEEN ENCOUNTERED WITH PROBUCOL IN RHESUS MONKEYS FED AN

Prolongation of the QT interval can occur in patients on Lorelco. Serious arrhythmias have been seen in association with an abnormally long QT interval in patients on Lorelco alone and in patients on Lorelco Patients should be advised to adhere to a low cholesterol, low fat diet at the start of treatment with Lorelco and throughout the treatment period

2. An ECG should be done prior to starting treatment and repeated at appropriate intervals during treatment. If an abnormally
long QT interval is observed, the possible benefits and risks should be carefully considered before making a decision to Lorelco therapy should be discontinued or not started if the OT interval at an observed heart rate on a resting ECG is

QT Interval in sec $15 \%$ above the

\begin{tabular}{|c|c|c|}
\hline \multirow{2}{*}{$\begin{array}{l}\text { Observed Heart Rate } \\
\text { (beats/min) }\end{array}$} & \multicolumn{2}{|c|}{ upper limit of normal) } \\
\hline & Males & Females \\
\hline $\begin{array}{r}40 \\
50 \\
60 \\
70 \\
80 \\
86 \\
92 \\
100 \\
109 \\
120 \\
133\end{array}$ & $\begin{array}{l}0.56 \\
0.52 \\
0.49 \\
0.45 \\
0.43 \\
0.42 \\
0.40 \\
0.39 \\
0.37 \\
0.36 \\
0.34\end{array}$ & $\begin{array}{l}0.58 \\
0.53 \\
0.50 \\
0.47 \\
0.44 \\
0.43 \\
0.41 \\
0.40 \\
0.38 \\
0.36 \\
0.35\end{array}$ \\
\hline
\end{tabular}

"Values calculated trom Burch GE, Winsor T. A primer of electrocardiography. Philadelphia, PA: Lea and Febiger, 1958; P.

3. Patients developing unexplained syncope or syncope of cardiovascular origin should have Lorelco therapy discontinued and

should have ECG surveillance.

4. Drugs that prolong the $O T$ interval are more likely to be associated with ventricular tachycardia after:

a. An increase in the dose of the drug.
b. Addition of a second drug that prolongs the QT interval (including tricyclic antidepressants, class I and III antiarritythmics,

. Hypokalemia or hypomagnesemia.

d. Severe bradycardia due to intrinsic heart disease or drug effects on the atrial rate (beta-blockers) or AV block (digoxin)

The use of Lorelco in patients receiving any of these drugs should be based on the conclusion that tial benefits of cholesterol lowering outweigh the risk of serious arrhythmia.

The following conditions should be resolved or corrected prior to initiation of therapy with Lorelco:

a. Hypokalemia

Syeverragnesemia bradycardia due to intrinsic heart disease or drug effects on the atrial rate (beta-blockers) or AV block (digoxin). PRECAUTIONS General: Before instituting therapy with Lorelico, adequate baseline studies should be performed to determine that the patien
has persistently elevated fotal and LDL-cholesterol levels representing a primary lipid disorder, and that the increased
cholesterol is not due to secondary conditions such as hypothyroidism, poorly controlled diabetes mellitus, obstructive liver disease, nephrotic syndrome, or dysproteinemias. Serum lipid levels, including HOL-cholesterol, should be determined after overnight fast before treatment, during an adequate trial of diet and weight reduction therapy prior to addition of drug therapy.
and periodically during combined diet and drug therapy. including assessment during the first several months of drug treatment. and periodically during combined diet and drug therapy, including assessment during the first several months of drug treatment
A favorable trend in lipid levels should be evident during the first three to four months of administration of Lorelco, and
satisfactory lipid alteration is not achieved, the drug should be discontinued. Lorelco lowers serum total and LDL-cholesterol and also lowers $\mathrm{HDL}$-cholesterol in most patients with elevated $\mathrm{LDL}$-cholesterol. Epidemiologic studies within hypercholesterolemic populations have shown that serum HDL-cholesterol is an independent, inversely correlated, risk factor for coronary heart disease (see CLINICALPHARMACOLOGY). Human studies which will attempt to confirm or deny the hypothesis
that drug-induced alteration in HOL-cholesterol affects cardiovascular risk are currently under evaluation. It is not known that drug-induced alteration in
whether Lorelico-induced reduction of serum HDL-cholesterol will affect careiovascular risk since no lon. It is not know
clinical trials of Lorelco for the controlled been pertormed. The probable benefits obtained from LDL-cholesterol reduction must be weighed againict the possible risk of a
reduction in HDL-cholesterol when assessing the response of each patient receiving Lorelco treatiment. If satisfactory lipid reduction in $\mathrm{HDL}$-cholesterol when assessing the response
alteration is not achieved, the drug should be discontinued.
Carcinogenesis, Mutagenesis, Impairment of Fertility

the lack of amy adverse effect on fertility and the negative findings in tests for mutagenic activity in rats.

Pregnancy
Teratogenic Effects

Peragnency - Category 8: Reproduction studies have been pertormed in rats and rabbits at doses up to 50 times the humar
Prese and have revealed no evidence of impaired fertility or harm to the fetus due to probucol. There are, however, no adequar
dose and well-controlled studies in pregnant women. Because animal teproduction studies are not always predictive of huma
response. response, this drug should be used during pregnancy only if clearly needed. Furthermore, if a patient wishes to becom persistence of the drug in the body for prolonged periods. (See CLINICAL PHARMACOLOGY.)

Nursing Mothers: It is not known whether this drug is excreted in human milk, but it is likely, since such excretion has bee Pediatric Use: Satety and effectiveness in children have not been established.

ADVERSE REACTIONS

Gastrointestinal
diarthea or loose stools, flatulence, abdominal pain, ılausea, vomiting, indigestion, gastrointestinal bleeding

Crordiovascular Neurologic

Hematologic

Dermatologic

Genitourinary

tearing, blurred vision

enlargement of multinodular goiter

Dosiosyncrasies
observed with initiation of therapy and characterized by dizziness. palpitations, syncope, nausea, vomiting and chest pa diminished sense of taste and smell, anorexia, angioneurotic edema

DRUG ABUSE AND DEPENDENCE: No evidence of abuse potential has been associated with Lorelco, nor is the OVERDOSAGE: There is a single report of a $15-\mathrm{kg}$, three-year-old, male child who ingested $5 \mathrm{~g}$ of probucol. Emesis war

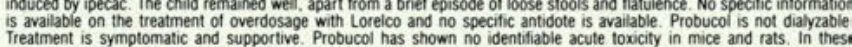
Treatment is symptomatic and supportive. Probucol has
animals the $L \mathrm{~L}_{0}$ (oral) is in excess of $5 \mathrm{~g} / \mathrm{kg}$ of body weight

DOSAGE AND ADMIINISTRATION: For adult use only. The recommended and maximal dose is $1000 \mathrm{mg}$ daily given (1)

$500 \mathrm{mg}$ capsule-shaped, white, film-coated tablets, marked LOREL C 500 . Bottles of 100 (NDC 0068-0053-61)

Keep well closed. Store in a dry place. Avoid excessive heat. Dispense in well-closed light-resistant containers with child ANIMAL PHARMACOLOGY AND TOXICOLOGY: In rhesus monkeys, administration of probucol in diets containin Unusually high amounts of cholesterol and saturated lat resulted in the death of four of eight animals after several weeks
Premonitory syncope was trequently observed and was associated with a pronounced prolongation of the QT intervals (30 to to
$50 \%$ longer than that observed in untreated monkeys). Serum levels of probucol greater than 200 mogimL were $50 \%$ longer than that observed in untreated monkeys. Serum levels of probucol greater than 20 mog/mL were generall:h
associated with some prolongation in the QT interval in the cholesterol-ted monkey. A 5 msec or greater increase in Qf interva from control values was usualily seen at $40 \mathrm{mcg} / \mathrm{mL}$ and above. Blood levels in humans receiving Lorelco average approximatel
$20 \mathrm{mcg} / \mathrm{mL}$ and not uncommonly reach levels of $40 \mathrm{mcg} \mathrm{mL}$ and higher. Rhesus monkeys ted normal (low fat chow ane receiving probucol three to thirty times the human dose equivalent achieved blood levels only one-third those of many huma
subjects. No adverse effects were detected in these monkeys over an eight-year period of continuous drug administration. another study in rhesus monkeys, an atherogenic diet was fed for two years and daily treatment with probucol, separated in tim

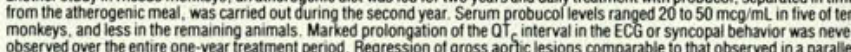
group of monkerys receiving cholestyramine was seen in animals receving probucol. It should be emphasized that both HDL cholesterol and DL-cholesterol were markedly reduced in this regression sto
study involving 32 probucol-treated dogs (beagles), there were 12 tatalities.

Subsequent experiments have indicated that probucol sensitizes the canine myocardium to epinephrine, resulting in ventricul fibrillation in many dogs. Among the animal species in which probucol has been studied, the dog is peculiar with respect to th
phenomenon of sudden death due to the sensitization of the myocardium to epinephrine. In contrast to findings in the dog

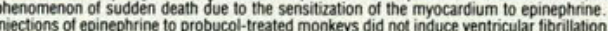

In other studies, monkeys were given probucol either before and atter, or only after myocardial intarction was induced b coronary artery ligation. In these studies, there was no difference between probucol-and placebo-tre
either survival or detailed blind quantitation of myocardial changes (gross and histopathologic).

Probucol has shown no identifiable toxicity in mice and rats. In these animals, the LDSo (oral) is in
weight. In chronic studies of two years' duration in rats, no toxicity or carcinogenicity was observed probucol administered orally is unabsorbed. For that why
the body and very little is excreted by way of the kidneys.

Myocardial injury was produced in various groups of rats by one of the following procedures: aortic coarctation, corona ligation, or cobalt or isoproterenol injection. After probucol administration, no
as measured by survival and microscopic examination of myocardial damage.

Probucol was administered to minipigs beginning ten days before ligation of coronary artery and continued for 60 days atte surgery Challenge with ed
probucol-treated minipigs.

CLINICAL STUDIES: In a multicenter, randomized, double-blind study, the LRC-CPPT, ${ }^{3}$ hypercholesterolemic patient treated with an oral bile acid sequestrant (cholestyramine) and a cholesterol-lowering diet experienced average total and $L D$
cholesterol reductions greater than those obtained in the placebo group treated with diet alone. The cumulative seven-yea incidence of the primary end point-Combined incidence of definite $C H D$ death and/or definite nonfatal myocardial infarction-
was $7 \%$ in the cholestyramine group and $8.6 \%$ in the placebo group. This was a $19 \%$ reduction in risk (P less than 0.05 , single tail test) of
intarction.

The subjects included in the study were middle-aged men (35-59 years oid) with serum cholesterol levels at least 265 myddt. an no previous history of heart disease. It is not clear to what extent these findings can be extrapolated to other segments of

The bile acid sequestrant, cholestyramine, was used in the above trial. Caution should be exercised in extrapolating these result:

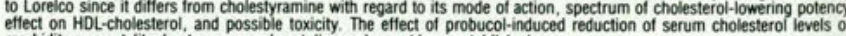
REFERENCES

1. Consensus Development Panel. Lowering blood cholesterol to prevent heart disease. JAMA. 1985: 253:2080-2086. 3. The Lipid Research Clinics Progran. The Lipid Reserch Clinics coronary primary prevention trial results: I. Reduction Product Information as of June, 198

MERRELL DOW PHARMACEUTICALS INC.

Subsidiary of The Dow Chemical Company

\section{Merrell Dow}

Ophthaimic 


\section{JAOA CME QUIZ}

FILL OUT AND RETURN THIS CARD FOR CME CREDIT (1 HOUR):

American Osteopathic Assn., Attn: Div. of CME, 142 E. Ontario St., Chicago, IL 60611-2864

"Certification of Home Study" form need not be submitted with this quiz.

Member number 00 (see membership card)

1. $\mathrm{a} b$

2. $a b c d e$

3. $a \mathrm{~b} c \mathrm{~d}$ e

4. $\mathrm{a} b \mathrm{c}$
5. a b

6. $\mathrm{a} \mathrm{b}$

7. $\mathrm{a} \mathrm{b}$

8. a b c d
9. $a b c d$

10. a b c d

11. a b c d

12. a b 
American Osteopathic Association Attn: Division of CME 142 East Ontario Street Chicago, IL 60611-2864 
stimulate renewed resolve of our osteopathic colleges and to encourage them to prepare through the formulation and implementation of their own AIDS policies.

\section{FDA: FDA Drug Bulletin.}

2. Harris, CE: AIDS and the future. Healing Ethic. 2:1.

3. The AIDS Crisis in New York: The Role of New York's Medical Schools and Academic Health Centers. New York, Associated Medical Schools of New York, 1987.

4. Recommendations for prevention of HIV transmission in health-care settings.MMWR 1987:36 (suppl 2S); 3S-18S.
5. Revision of the CDC surveillance case definition for acquired immunodeficiency symdrome. MMWR 1987;36(suppl to No. 1):35-155.

6. Report of the Ohio Osteopathic association ad hoc committee on AIDS: Presented to the State Medical Board of Ohio, Columbus, Ohio, October $15,1987$.

From the Department of Family Medicine Ohio University College of Osteopathic Medicine, Athens, $\mathrm{OH} 45701$.

Dr Duerfeldt, OU-COM, Grosvenor Hall Athens, OH 457012979 